\title{
Turismo e Transnacionalização da Intimidade nos Trópicos Globais
}

\author{
Tourism and Intimacy Transnationalization in the Global Tropics
}

\author{
Turismo y Transnacionalización de la Intimidad en los Trópicos Globales
}

Octávio Sacramento ${ }^{1}$

\begin{abstract}
Resumo
O texto proporciona um breve mapeamento dos destinos tropicais à escala global onde o turismo de massas de sol e praia se entrecruza com a constituição de relacionamentos íntimos transnacionais. Considerando as experiências turísticas e as configurações de intimidade que aí emergem, o objetivo prioritário da reflexão passa por questionar a pertinência da noção de turismo sexual como conceito hegemónico na generalidade dos exercícios de análise e conceptualização destes contextos. Embora se reconheça a presença de fortes expectativas passionais associadas às mobilidades turísticas para muitos destinos da cintura tropical global, tenta estabelecer-se um distanciamento crítico face à ideia de turismo sexual. O intuito é mostrar que se trata uma designação que, além de enredada em estereótipos, denota uma grande inconsistência epistemológica e é profundamente redutora, incapaz de traduzir a complexidade e o dinamismo subjacentes ao quadro de expectativas, práticas e relações que configuram os encontros íntimos transnacionais entre visitantes e locais.
\end{abstract}

Palavras-chave: Turismo sexual; Intimidades transnacionais; Trópicos globais.

\begin{abstract}
The text provides a brief global mapping of the tropical destinations where the sun and beach mass tourism is connected with the constitution of intimate transnational relationships. Considering the touristic experiences and the intimacy configurations that emerge there, the main objective of the reflection is to question the pertinence of the sex tourism notion as hegemonic concept in almost all exercises of analysis and conceptualization of these contexts. Although recognizing the presence of strong passional expectations linked to the touristic mobility for many destinations of the global tropical belt, a critical distance to the idea of sex tourism is established. The aim is to show that this designation is entangled in stereotypes, denotes a great epistemological inconsistency and is deeply reductive, unable to translate the complexity and the dynamics underlying the framework of expectations, practices and relationships that constitute the transnational intimate meetings between visitors and locals.
\end{abstract}

Keywords: Sexual tourism; Transnational intimacies; Global tropics.

\footnotetext{
${ }^{1}$ Doutor em Antropologia pelo Instituto Universitário de Lisboa (ISCTE-IUL), Portugal. Mestre em Sociologia da Cultura e dos Estilos de Vida pela Universidade do Minho, Portugal. Licenciado em Antropologia Social e Cultural pela Universidade Nova de Lisboa (UNL), Portugal. Investigador integrado do Centro de Estudos Transdisciplinares para o Desenvolvimento (CETRAD-UTAD). Professor auxiliar da Universidade de Trás-osMontes e Alto Douro; Departamento de Economia, Sociologia e Gestão (UTAD-DESG) Vila Real, Portugal. Email: octavsac@utad.pt
} 


\section{Resumen}

El texto proporciona un breve mapeo de destinos tropicales a nivel mundial donde el turismo de masas de sol y playa se cruza con la formación de relaciones íntimas transnacionales. Considerando las experiencias turísticas y las configuraciones de intimidad que ahí emergen, el objetivo prioritario de la reflexión pasa por cuestionar la pertinencia de la noción de turismo sexual como concepto hegemónico en la mayoría de los ejercicios de análisis y conceptualización de estos contextos. Aunque reconociendo la presencia de fuertes expectativas pasionales ligadas a la movilidad turística para muchos destinos de la cintura tropical global, se establece una distancia crítica ante la idea de turismo sexual. El objetivo es mostrar que esta es una designación que, además de enredada en estereotipos, denota una gran incoherencia epistemológica y es profundamente reductiva, incapaz de traducir la complejidad y la dinámica subyacentes a las expectativas, prácticas y relaciones que constituyen los encuentros íntimos transnacionales entre visitantes y locales.

Palabras clave: Turismo sexual; Intimidades transnacionales; Trópicos globales.

\section{Introdução}

Os fluxos populacionais globais, mesmo os mais efémeros, produzem contactos entre pessoas de diferentes nacionalidades e proporcionam-lhes possibilidades de constituição de espaços sociais de convivência íntima que extravasam múltiplas fronteiras, nomeadamente as fronteiras político-administrativas que delimitam os Estados-nação. Esta "transnacionalização da intimidade"2 está presente em muitos destinos internacionais de lazer, sobretudo em contextos tropicais de turismo balnear de massas do Sudeste asiático e da América Latina. De um modo geral e recorrente são considerados cenários de turismo sexual, dando-se a entender que se tratará de mais um tipo distinto de turismo. Tal acontece não só na esfera do senso comum e do espaço mediático, como também no meio académico. Neste último, a designação de turismo sexual tende a ser assumida como um conceito de facto, sendo que a sua amplitude epistemológica e sustentabilidade empírica raramente são questionadas.

\footnotetext{
${ }^{2} \mathrm{O}$ termo surge timidamente, sem grande desenvolvimento conceptual, num texto de King (2002) sobre novas tipologias e geografias das migrações internacionais na Europa. Através dele, o autor chama a atenção para a crescente relevância de factores pessoais e sentimentais na ocorrência de fluxos migratórios no continente europeu. Desde então tem sido usado sobretudo no estudo das chamadas "migrações por amor" (Roca, 2009). Tomo-o aqui de empréstimo e amplio o seu alcance, procurando com ele traduzir as relações afectivo-sexuais e matrimoniais à escala internacional decorrentes das mobilidades turísticas.
} 
Ainda que de uma forma muito sumária, é justamente este questionamento que aqui se procura esboçar, tentando evidenciar que a noção de turismo sexual é simplista e redutora ao sugerir a existência de uma motivação sexual estrita a impulsionar a procura turística. Aliás, a sua utilização como suposto conceito científico pressupõe, geralmente, a sobrevalorização explicativa desta motivação (BEM, 2005; BRENNAN 2004a; ENLOE, 2000; GRABURN, 1983) e a respectiva associação ao género masculino, pelo que "the stereotypical image of the 'sex tourist' is that of the Western man who travels to Thailand or the Philippines in order to pay for sex with Go Go bar/brothel prostitutes" (TAYLOR, 2001: 749). Alegadamente, estes homens apenas querem comprar sexo como se de uma mercadoria se tratasse (MICHEL, 2009) e as mulheres que com eles se relacionam apenas querem assegurar renda com a venda do seu corpo, num jogo que se resumiria ao "poder do dinheiro" versus o "poder do sexo", para usar os termos de Lagunas (2010).

Se o turismo para os chamados destinos sexuais representasse somente viagens de homens à procura de sexo esporádico, como compreender as situações de envolvimento duradouro e os inúmeros projectos conjugais e familiares, ainda que, em muitos casos, a sua génese se situe no contexto da prostituição (COHEN, 1982, 2003; KEMPADOO, 1999; PISCITELLI, 2007)? Para lá do sexo, da parte masculina, e do dinheiro, da parte feminina, existem muitas outros desejos, desígnios e interesses de parte a parte (CABEZAS 2004; PISCITELLI, ASSIS E OLIVAR, 2011; SACRAMENTO, 2015a,b) - associados, por exemplo, ao género, à aliança, à família e às migrações - que importa considerar. Assim é possível evitar visões imediatistas, atadas à suposição de que tudo se resume à mera contratualidade económico-sexual induzida pela voragem mercantil do turismo de massas.

As reflexões aqui desenvolvidas, embora panorâmicas e de perfil teórico-conceptual, decorrem do trabalho de investigação para doutoramento realizado pelo autor (SACRAMENTO, 2014) ${ }^{3}$, no âmbito do qual procurou apreender as relações de intimidade entre turistas europeus e mulheres brasileiras durante as estadias dos primeiros no bairro balnear de Ponta Negra (Natal-RN, Nordeste brasileiro), bem como a evolução das suas aproximações passionais para determinadas formas de conjugalidade e os fluxos transatlânticos aí implicados. Perante os elementos empíricos proporcionados pela etnografia, principal estratégia metodológica utilizada na pesquisa de terreno, foi possível constatar as

\footnotetext{
${ }^{3}$ Esta investigação beneficiou de uma bolsa de doutoramento da Fundação para a Ciência e a Tecnologia de Portugal (SFRH/BD/60862/2009).
} 
incoerências e insuficiências conceptuais e a difícil operacionalização da noção de turismo sexual como ferramenta analítica. Em texto anterior (SACRAMENTO, 2011) já se identificavam, aliás, muitas das suas possíveis limitações e imprecisões epistemológicas.

\section{Trópicos Turísticos Passionais}

A formação dos grandes impérios ultramarinos estabeleceu hierarquias geopolíticas e económicas transnacionais, e instaurou uma densa colonização simbólica e epistemológica dos trópicos (MIGNOLO, 2000), ${ }^{4}$ contribuindo, assim, para a sua génese como eminentes geografias de lazer, prazer e erotismo (MCCLINTOCK, 1995). Foi, justamente, nas reminiscências da antiga ordem colonial, bem como nas suas imagens e imaginações do "outro", carregadas de atribuições exóticas, sensuais e sexuais - entretanto apropriadas e (re)modeladas nos processos pós-coloniais de afirmação das identidades nacionais (MACHADO, 2009; SOMMER, 2004) -, que começaram a estruturar-se muitas das aspirações, desejos e seduções interculturais que impulsionam as configurações transnacionais de intimidade. Aliás, a expansão do turismo de massas nas periferias tropicais, e não só, é ela própria largamente tributária de ordenamentos internacionais, desígnios de exotismo e genuinidade, e fascínios nostálgicos edificados sob o legado dos impérios coloniais (D'HAUTESERRE, 2008; JAACKSON, 2004; PISCITELLI, 2004d; TURNER E ASH, 1991). O processo de produção e internacionalização turística, ainda que nem sempre de forma intencional, favoreceu o "male gaze" (PRITCHARD E MORGAN, 2000) e reproduziu as lógicas do desejo (CONSTABLE, 2003) dos "fantasmas do império" (VEISSIÈRE, 2011), transformando a intimidade em objecto das aspirações turísticas.

Embora se possa admitir que a associação entre o turismo e a transnacionalização da intimidade existe em diferentes latitudes, incluindo na Europa e na América do Norte (OPPERMANN, 1999), quase todos os estudos sobre esta temática são realizados nos "pornotrópicos" (MCCLINTOCK, 1995) dos antigos impérios coloniais, os "sítios de desejo" (MANDERSEN E JOLLY, 1997) mais destacados à escala mundial. Temos, assim, bastante pesquisa já desenvolvida em cidades do Nordeste brasileiro, como Natal-RN (CANTALICE, 2009a, 2009b; CARRIER-MOISAN， 2008; RIBEIRO E SACRAMENTO, 2006; SACRAMENTO E RIBEIRO, 2013; SACRAMENTO 2014, 2015), Fortaleza (BEZERRA,

\footnotetext{
${ }^{4}$ De um modo geral, os processos de independência nacional não reverteram de forma verdadeiramente
} significativa esta colonização simbólica e algumas das subalternidades das ex-colónias à escala global. 
2010; PISCITELLI, 2004a, 2004b, 2007), Recife (CARPAZOO, 1994), Salvador da Bahia (FILHO, 1998; FILHO E SARDENBERG, 1998; MARQUEZ, 2009) e, mais para sul, no Rio de Janeiro (BLANCHETTE E SILVA, 2010; SILVA E BLANCHETTE, 2005). Na restante América Latina tropical são de relevar as pesquisas realizadas na área do Caribe, nomeadamente em Cuba, na República Dominicana e na Jamaica (BRENNAN 2001a,b, 2004a, 2004b; CABEZAS, 2004; FROHLIC, 2008; KEMPADOO, 1999; O'CONNELLDAVIDSON, 1996; PRUITT E LAFONT, 1995; SIMONI, 2011; TAYLOR, 2001). Do outro lado do Oceano Pacífico, o Sudeste asiático é, já há muito, uma conhecida geografia turística passional (BISHOP E ROBINSON, 1998; COHEN, 1982; LEHENY, 1995; LEUNG, 2003; O'CONNELL-DAVIDSON，1995; OPPERMANN E MCKINLEY，1997; RAO，1999; TRUONG, 1983, 1990). Aqui destacam-se as Filipinas e, acima de tudo, a Tailândia, que, desde a presença militar americana na década de 1960, se transformou numa espécie de capital sexual global (COHEN, 2003). Em sentido oposto, o continente africano parece ainda não figurar como contexto cimeiro destes cenários, o que ajuda a explicar, de alguma forma, o facto de a investigação relativa a esta área não ser muito abundante. O Quénia é uma das poucas excepções (KIBICHO, 2009; OMONDI, 2003).

A instalação de bases dos Estados Unidos da América (EUA) no Sudeste asiático durante a guerra do Vietname, nomeadamente na Tailândia, foi acompanhada pelo aparecimento de restaurantes, bares, casas de massagens, hotéis, nightclubs e bordéis nas suas imediações (COHEN, 2003: 60-61). Desta ecologia, que Cohen (idem) designa por pleasure belt, resultavam diferentes tipos de relacionamentos, mais ou menos mercantilizados, entre os militares americanos e as mulheres tailandesas. Não se tratava simplesmente de prostituição. Era bastante frequente, por exemplo, a mesma mulher permanecer com o mesmo soldado durante a sua estadia no país, vivendo na mesma casa como se de uma esposa se tratasse. Era a chamada rented wife. Algumas destas relações culminaram em casamento e, na maior parte dos casos, implicaram a ida do casal para os EUA. A presença do exército norte-americano na Tailândia contribuiu, assim, para a disseminação global da imagem do país como destino sexual, à semelhança do que sucedeu noutros contextos de acolhimento de bases militares (ENLOE, 2000). Também no Nordeste brasileiro, na cidade de Natal-RN, em plena Segunda Guerra Mundial, foram instaladas bases americanas com um contingente total na ordem de 10.000 homens (JÚNIOR, 1993), o que alterou significativamente a configuração do mercado passional local. Dessa época permanece ainda bem viva na memória colectiva da cidade a 
figura de Maria Boa (Maria Barros), dona de um grandioso cabaré que tinha como principais clientes as altas patentes militares norte-americanas. Ainda sobre o impacto das deslocações militares massivas na organização social da intimidade, Min (2003) descreve-nos como o Japão, durante a guerra da Ásia e Pacífico (1937-1945), mobilizou cerca de 200.000 jovens mulheres da Coreia (na altura colónia japonesa) para os bordéis das bases militares que haviam sido estabelecidas na China e em demais países do Sudeste asiático. Estas mulheres ficaram conhecidas como comfort women. Em 1999 tornaram-se "objecto turístico" com a criação do Comfort Women Museum, nos arredores de Seul, na Coreia do Sul (YEA, 2003).

Em contraste com o Sudeste asiático, a inserção da América Latina e, mais em concreto, do Nordeste brasileiro, em circuitos mundiais generificados e sexualizados de turismo terá sido mais recente. Recorrendo à análise dos conteúdos dos sites de viajantes à procura de sexo, Piscitelli (2005) constata que essa inserção tornou-se particularmente evidente em finais da última década do século XX. Apesar das suas especificidades, quase todos estes destinos têm em comum a presença conjugada dos quatro S's do marketing turístico - sun, sand, sea, sex (LOWRY, 1993) - e, de um modo geral, são frequentados maioritariamente por turistas masculinos heterossexuais. Todavia, a presença de turistas mulheres nos mesmos lugares ou em contextos diferenciados, envolvendo-se com homens locais, começa também a ganhar alguma expressão (CANTALICE 2009a,b; FROHLICK 2008a,b; HEROLD, GARCIA E DEMOYA, 2001; PHILLIPS, 1999, 2008; TAYLOR, 2001), bem como o turismo gay (CLIFT, CALliSTER E LUONGO, 2002; COLLINS, 2007). No entender de Phillips (2008), o envolvimento feminino nestes fluxos passionais remonta à década de 1960, numa altura em que mulheres inglesas, alemãs e escandinavas se deslocavam em férias para o sul da Europa. Com a massificação do turismo terão começado a viajar para destinos mais distantes.

Quando se consideram cenários sociais como os que têm vindo a ser identificados ao longo da cintura tropical global, onde o turismo se entrecruza com a prostituição e outras formas de relacionamento íntimo entre visitantes e locais, a noção de turismo sexual é, incontornavelmente, utilizada pelas ciências sociais - também pelo senso comum e pelo espaço mediático - como uma espécie de frame dos seus discursos. Desta forma, parece estar a identificar-se mais um tipo distinto de turismo, como o cultural, o balnear ou o rural. Até se poderá compreender que assim seja no domínio do senso comum e dos discursos mediáticos. Mais estranho é quando o meio académico se apropria da designação de forma acrítica, não questionando a imensa carga de estereótipos, moralismos e pressupostos ideológicos que 
transporta. Alguns autores reconhecem, no entanto, que se trata de um conceito bastante problemático (PISCITELLI, 2004b; TAYLOR, 2001) e de difícil operacionalização. A principal razão para tal advém do facto de apresentar uma amplitude semântica incerta e uma grande volatilidade de sentidos, sendo usado para traduzir uma grande diversidade de configurações sociais em que o turismo e a expressão da intimidade estão relacionados. Impõe-se, por isso, que seja submetido a um exercício efectivo de precisão conceptual e de crítica epistemológica (SACRAMENTO, 2011).

Numa perspectiva francamente ampla, a noção de turismo sexual tende a ser utilizada para fazer referência à circulação de pessoas, em tempo de lazer, que tem por base expectativas sobre possíveis relacionamentos afectivo-sexuais, heterossexuais ou homossexuais, de natureza comercial ou não, com outras pessoas que se encontram nos destinos turísticos (CABEZAS, 2004; COHEN, 1986; MCKERCHER E BAUER, 2003; OPPERMANN, 1999). Aqui poderá enquadrar-se um conjunto bastante diversificado de situações, desde um extremo em que as relações evidenciam uma dimensão comercial, associada ou não à exploração e à coerção, podendo envolver crianças e jovens, até um outro caracterizado por relacionamentos não comerciais e voluntários entre adultos (RYAN, 2000: 35-36). A par deste entendimento amplo existem visões mais circunscritas e estereotipadas, segundo as quais o turismo sexual corresponde a situações em que a experiência turística se entrecruza com a prostituição e a exploração sexual de mulheres e crianças, tendo subjacente uma estrutura de género que traduz o poder do homem sobre a mulher (ENLOE, 2000; GRABURN, 1983; HALL, 1996; RAO, 1999; TRUONG, 1990). Nesta perspectiva, Jeffreys (in TAYLOR, 2001: 749) defende que a designação mais adequada deveria ser "prostitution tourism", destacando que o fenómeno resulta de uma socialização masculina ocidental na qual o uso comercial de mulheres para auto-satisfação sexual é tido como um direito natural da masculinidade.

Independentemente das suas diferentes acepções e utilizações, em regra o alegado conceito tem sido empregue para traduzir as mobilidades de lazer de homens dos países ricos do hemisfério norte para determinados países pobres do hemisfério sul, onde acedem a relações sexuais com mulheres que se prostituem. Na argumentação de Enloe (2000: 36), o turismo sexual resultaria da convergência das seguintes circunstâncias: (i) conjugação de interesses entre as autoridades governamentais locais, ansiosas por assegurar novas fontes de renda, e o investimento estrangeiro no sector do turismo, disposto a apostar em viagens com uma forte componente sexual; (ii) mulheres economicamente desesperadas ao ponto de se envolverem 
em relacionamentos mercantis com os turistas; (iii) homens de sociedades afluentes à procura do que julgam ser mulheres mais submissas que as dos seus países de origem. As assimetrias materiais entre ambas as partes são identificadas como variável determinante de uma relaçãotransacção sexista e patriarcal em que a maior capacidade económica dos homens é invocada como o factor que, por si só, lhes garante uma posição dominante.

Quando o destino da mobilidade turística se situa num país desenvolvido do hemisfério norte deixa de ser tão consensual a utilização do conceito de turismo sexual, mesmo que o contexto seja fortemente sexualizado e acolha um grande número de mulheres pobres do Sul a trabalhar no mercado do sexo, como é o caso de Amesterdão ${ }^{5}$. Ao que parece, só haverá verdadeiramente turismo sexual abaixo do equador, nos trópicos, sendo o fenómeno apresentado, amiúde, como mais uma expressão neocolonialista (BEM, 2005; GRABURN, 1983; KRIPPENDORF, 1989; OURIQUES, 2005). Por outro lado, a utilização do conceito e a sua associação ao neocolonialismo parece só fazer sentido quando estamos em presença de turistas heterossexuais do sexo masculino. Ao se inverter o género dos intervenientes, ou seja, mulheres do Norte e homens do Sul, o uso do conceito deixa de ser tão taxativo. Geralmente é substituído por "turismo de romance" e os locais que se envolvem com as turistas designados por beach boys ${ }^{6}$ (HEROLD, GARCIA E DEMOYA, 2001; PHILLIPS, 1999). Alega-se que eles desempenham um papel de mediadores culturais (BROWN, 1992) e que a relação assenta sobretudo na afectividade e não tanto numa contratualidade económica estrita (CANTALICE, 2009a; DAHLES, 2002; DAHLES E BRAS, 1999; PRUITT E LAFONT, 1995) ${ }^{7}$. Nestas circunstâncias já não haverá prostituição nem opressão, simplesmente novas oportunidades de negociação e expressão das identidades de género (DAHLES, 2002). De igual modo, parece perder cabimento a tese da exploração neocolonial.

\footnotetext{
${ }^{5}$ Wonders e Michalowski (2001) destoam desta tendência ao considerarem Amesterdão um cenário de turismo sexual, analisando-o no mesmo texto, lado a lado, com Havana.

${ }^{6}$ Quer numa perspectiva emic (a das próprias comunidades) quer numa perspectiva etic (a do meio académico), as designações "turismo de romance" e beach boys são manifestamente neutras quando comparadas com as que são utilizadas quando se trata de mulheres locais e homens estrangeiros. Considerando que os discursos constituem os objectos de que falam, estas nuances terminológicas parecem expressar a persistência de um duplo padrão moral de controlo da sexualidade feminina, que tolera ou até valoriza o envolvimento sexual dos homens com as turistas, mas que estigmatiza os comportamentos sexuais femininos equivalentes, rotulando as intervenientes de prostitutas ou trabalhadoras sexuais.

${ }^{7} \mathrm{O}$ mesmo argumento é utilizado por Collins (2007) para o turismo gay, destacando que a prestação de serviços sexuais homossexuais se trata de um "trabalho desejado".
} 


\section{Questionando Perspectivas Monetaristas e Sexocêntricas}

O estereótipo do turista sexual como homem, ocidental e afluente, viajando para países pobres com o estrito propósito de se envolver sexualmente com mulheres vulneráveis revela componentes ideológicos e alguns enviesamentos que importa considerar: (i) a utilização da terminologia turismo sexual ou turismo de romance em função do género dos turistas parece estar contaminada pela tradicional ideologia sexista, assente em valores totalitários e essencialistas do masculino e do feminino, segundo os quais os homens procuram sexo numa relação, enquanto as mulheres procuram afecto e romance; (ii) o poder monetário masculino e a mercantilização feminina da sexualidade, principais referentes da ideia de exploração, deixam de ser utilizados quando os papéis de género se invertem, como se as assimetrias económicas fossem completamente inócuas quando pendem a favor da mulher; (iii) as concepções culturais hegemónicas do masculino como activo e dominante e do feminino como passivo e dominado (PARKER, 1991) parecem constituir a principal matriz simbólica dos discursos que identificam o turismo sexual (masculino) como reencarnação da antiga dominação colonial.

Referindo-se à existência de um duplo padrão de categorização do comportamento sexual de homens e mulheres turistas, Taylor (2006) conclui que tal reflecte as debilidades de muitos discursos científicos sobre género e poder, exploração sexual, prostituição e turismo sexual. Além dos enviesamentos de pendor sexista, da carga moral que geralmente comporta e de uma certa desconsideração pela capacidade de agência feminina, a noção de turismo sexual, como já foi dito, evidencia flutuações de sentido bastante vincadas e tem associados múltiplos paradigmas (RYAN, 2000; SACRAMENTO, 2011). Importa questionar, por isso, a pertinência da sua utilização no estudo das manifestações transnacionais de intimidade em cenários turísticos, tentando assim precaver a ideia reducionista de que o sexo mercantil é o móbil exclusivo e determinante da afluência turística. No caso mais comum dos turistas masculinos poder-se-á admitir-se que, de algum modo insatisfeitos com as manifestações dominantes de feminilidade e com as relações de género nos seus contextos de origem (O'CONNELL-DAVIDSON, 1995; SACRAMENTO, 2015a), procurem envolver-se com mulheres que lhes proporcionem vivências de intimidade mais condizentes com os seus ideais do que é "ser homem" e possam, desse modo, experienciar um certo rejuvenescimento viril (KRUHSE-MOUNTBURTON, 1995). Contudo, importa não esquecer que "the vast 
majority of tourists who use prostitutes to satisfy their sexual needs do not travel for that purpose alone" (OPPERMANN, 1999: 252). Muitas das suas motivações e práticas são comuns a qualquer outro "tipo" de turista e o sexo, seja comercial ou não-comercial, seja com locais ou com outros turistas, é quase sempre um elemento integrante e incontornável da experiência turística (MCKERCHER E BAUER, 2003).

Como justamente lembram Ryan e Kinder (1996: 507), “[...] tourists who go to sex workers are not simply just tacking on an activity to their tour, but are engaging in a fulfilment of types of motivations which are consistent with needs met by other forms of tourism". Estes autores, tal como Ryan e Hall (2001), encontram mesmo algumas semelhanças na condição "marginal" de turistas e trabalhadoras sexuais e um forte paralelismo entre as motivações da procura da prostituição e as motivações da mobilidade turística, nomeadamente as expectativas de ingressar num estado de liminaridade (TURNER, 1974), de quase-fantasia, que assegure contrastes catárticos face à vida de todos os dias. De facto, se considerarmos que a generalidade dos turistas procura situações novas, relaxamento, diversão, aventura e excitação - vivências liminóides (GRABURN, 2004); turismo como transição pessoal (NASH, 2001) - facilmente se percebe que estamos na presença de desígnios que também podemos identificar no fenómeno da procura da prostituição (RIBEIRO ET AL., 2007) e, de forma ainda mais explícita, na procura de relações de intimidade no quadro da própria experiência turística.

Além dos interesses sexuais masculinos e dos interesses económicos femininos existem muitas outras expectativas subjacentes à transnacionalização da intimidade no turismo que devem ser tidas em conta, sob o risco de se incorrer em simplificações e reducionismos. Numa análise da prostituição orientada para os turistas na Tailândia, Cohen (1982, 2003) mostra-nos a impossibilidade de se estabelecer uma fronteira nítida entre relações comerciais e relações amorosas, destacando as suas inúmeras ambiguidades e continuidades. Uma boa parte dos casamentos entre mulheres tailandesas e homens ocidentais representa o culminar de um processo em que a partilha de intimidade evolui da prostituição para o matrimónio. No entender de Cohen (idem), pode mesmo considerar-se a união conjugal com o turista (e posterior emigração) como a consequência última do exercício do sexo comercial. Neste caso, como realça Brennan (2001b) para a República Dominicana, a prostituição assume o papel de "stepping stone" para a maioria dos fluxos migratórios femininos. Encontramos situações 
bastante semelhantes na generalidade dos contextos turísticos que são considerados destinos sexuais globais (PISCITELLI, 2004c).

O casamento com o estrangeiro é uma aspiração que não se restringe às trabalhadoras sexuais. Ela está presente em muitas outras mulheres que não se assumem e/ou não são tidas como tal, muito em particular entre as mulheres das classes desfavorecidas. No entanto, nos contextos de lazer, como são as primeiras que estão socialmente mais acessíveis aos turistas, compreende-se o facto de ser mais frequente a ocorrência de relações mais prolongadas e até mesmo de casamentos como "derivados" do exercício da prostituição. Porém, são bastantes os relacionamentos e os matrimónios transnacionais associados às viagens de lazer masculinas que não têm a sua génese no meio prostitucional, mas sim no quadro mais geral das condições, circunstâncias e sociabilidades proporcionadas pela indústria do turismo (McKercher e Bauer, 2003: 12). Trata-se aqui de sociabilidades com mulheres que integram o grande contingente de mão-de-obra feminina do sector (v.g. funcionárias de hotéis, de restaurantes, de agências de viagens, de lojas de lembranças, vendedoras informais) e, menos frequente, com mulheres nacionais que também se encontram em turismo. A ecologia turística, na qual se pode incluir a prostituição, funciona como um ambiente social manifestamente propício à constituição de formas de intimidade que, por vezes, evoluem no sentido da conjugalidade.

Ao propiciar múltiplos idiomas e práticas relacionais (SIMONI, 2009), o turismo de massas é responsável pelo desenvolvimento de redes sociais de alcance transnacional que assumem um papel relevante na concretização de novos fluxos, sobretudo migratórios, protagonizados por cidadãos de países pobres. Muitas destas manifestações de "transnationalism from below" (SMITH E GUARNIZO, 1998) assentam numa densa malha de vínculos na qual se destaca a intimidade como elemento nuclear e centrípeto. Aliás, com a crescente fortificação das fronteiras dos Estados mais ricos, a intimidade, sobretudo quando conduz ao casamento, representa um dos meios mais imediatos/eficazes de aquisição de capital social e económico para a construção de projectos migratórios com alguma sustentabilidade. Não é por acaso que o matrimónio com o turista surge aos olhos de muitas mulheres asiáticas e latino-americanas como a solução mais plausível, desejada e procurada para concretizar a vontade de emigrar (BRENNAN, 2001B; COHEN, 2003). A sua capacidade de agência nesse sentido é bastante significativa, o que lhes vale no Brasil a alcunha de caçadoras de gringos. Elas mobilizam recursos (v.g. erótico-sexuais e relacionais) e desenvolvem performances de paixão para 
activar e manter vínculos com os turistas, demonstrando uma grande habilidade na utilização dos novos meios tecnológicos de comunicação para sustentar a relação à escala transnacional, depois do regresso dos seus parceiros aos respectivos países, e para a orientar na direcção do casamento. Não quer isto dizer que haja aqui, necessariamente, um calculismo estrito e uma mera instrumentalização da conjugalidade como forma de assegurar a deslocação migratória ou outros proveitos. De parte a parte, o amor assume muitos sentidos e a aliança pode, em simultâneo, ter subjacentes múltiplos interesses - incluindo aqui os de ordem passional e material - e prestar-se a diferentes usos e finalidades (BRENNAN, 2004a; SACRAMENTO, 2014).

\section{Conclusão}

O hemisfério sul ex-colonial é alvo de representações erotizadas baseadas em vínculos, imaginários e desejos antigos, alimentando uma "nostalgia imperial" (ROSALDO, 1989) na qual se inscrevem muitos dos actuais fluxos turísticos com destino aos trópicos. Apesar de se reconhecer a presença de expectativas erótico-sexuais e, inclusive, conjugais associadas a estas mobilidades, procurou vincar-se ao longo do texto um distanciamento crítico face à noção de turismo sexual, comummente utilizada como referência conceptual para designar as situações em que o turismo se entrecruza com a prostituição e com outras formas de relacionamento íntimo entre visitantes e locais. Tal opção ficou a dever-se a razões que remetem para os enredos morais e ideológicos em que o suposto conceito tende a ser envolvido e para a sua escassa consistência epistemológica. O campo semântico a que está adstrito é de tal forma indefinido, flutuante e pluri-paradigmático (RYAN, 2000; TAYLOR, 2001) que pode ser utilizado como referencial analítico de manifestações empíricas tão distintas como, por exemplo, a exploração sexual infanto-juvenil e os relacionamentos, comerciais ou não, entre adultos com capacidade de autodeterminação. Além de que se trata de uma designação bastante redutora, pois os turistas não procuram somente o consumo comercial de "sexo pelo sexo" nas suas estadias de lazer.

Embora o sexo constitua um elemento incontornável, seria simplista, todavia, apontar as motivações sexuais como causa explicativa absoluta da viagem e referencial exclusivo da análise da experiência turística. Congregando em geometria variável distintos desejos, interesses e projectos, os turistas esperam encontrar nos trópicos manifestações autênticas de feminilidade (PISCITELLI 2004d) que crêem em extinção no Ocidente, intensas experiências 
homossociais e um ambiente que os resgate de quotidianos entorpecidos do hemisfério norte (SACRAMENTO, 2014, 2015). A busca de uma suposta autenticidade permite-nos compreender o quanto valorizam e procuram a normalidade dos relacionamentos fora do contexto mais mercantil do sexo comercial e a generalizada apreciação negativa do acto de pagamento, directo e explícito, como condição de acesso à intimidade feminina (idem). Se é simplista a ideia de turistas apenas interessados em sexo como mercadoria, igualmente o é a ideia de que as mulheres com quem se relacionam só ambicionam assegurar vantagens materiais. É evidente que os trajectos e as condições de vida da maior parte destas mulheres lhes impõem preocupações constantes com os meios de subsistência, mais vincadas, expressivas e hegemónicas nuns casos, mais dissimuladas noutros. No entanto, estas preocupações encontram-se, invariavelmente, articuladas com muitas outras expectativas e projectos. Ao contrário do que é suposto quando se utiliza a ideia comum de turismo sexual, as demarcações entre o amor e o dinheiro são muito fluidas (ADELMAN, 2011), sendo bastante difícil estabelecer fronteiras inequívocas entre interesses afectivos, interesses materiais e outros interesses.

\section{Referências}

ADELMAN, M. Por amor ou por dinheiro? Emoções, discursos, mercados. Contemporânea: Revista de Sociologia da UFSCar, vol. 1, n. 2, p. 117-138, 2011.

BEM, A.S. A dialética do turismo sexual. Campinas: Papirus, 2005.

BEZERRA, R. 'Gringos e nativas': Representações do turista estrangeiro e suas acompanhantes no bairro Praia de Iracema na cidade de Fortaleza (Ceará-Brasil). In: SILVA, M.C. e RIBEIRO, F.B., eds. Mulheres da vida, mulheres com vida: Prostituição, Estado e políticas. Ribeirão: Edições Húmus, p. 205-220, 2010.

BISHOP, R. e ROBINSON, L. Night market: Sexual cultures and the Thai economic miracle. Londres e Nova Iorque: Routledge, 1998.

BLANCHETTE, T. e SILVA, A. P. 'A mistura clássica': Miscigenação e o apelo do Rio de Janeiro como destino para o turismo sexual. Bagoas, vol. 4, n. 5, p. 221-244, 2010.

BRENNAN, D. What's love got to do with it? Transnational desires and sex tourism in the Dominican Republic. Durham e Londres: Duke University Press, 2004a.

BRENNAN, D. When sex tourists and sex workers meet: Encounters within Sosúa, the Dominican Republic. In: GMELCH, S., ed. Tourists and tourism: A reader. Long Grove: Waveland Press, p. 303$315,2004 b$.

BRENNAN, D. Tourism in transnational places: Dominican sex workers and German sex tourists imagine one another. Identities: Global Studies in Culture and Power, vol. 7, n. 4, p. 621-623, 2001a. 
BRENNAN, D. Selling sex for visas: Sex tourism as stepping stone to international migration for Dominican women. In: EHRENREICH, B. e HOCHSCHILD, A., eds. Global women: Nannies, maids, and sex workers in the new economy. Nova Iorque: Metropolitan Books, p. 154-168, 2001b.

BROWN, N. Beachboys as cultural brokers in Bakau Town, the Gambia. Community Development Journal, vol. 27, n. 4, p. 361-370, 1992.

CABEZAS, A. Between love and money: Sex tourism and citizenship in Cuba and the Dominican Republic. Signs - Journal of Women in Culture and Society, vol. 29, n. 4, p. 987-1015, 2004.

CANTALICE, T. 'Dando um banho de carinho' - Os caça-gringas e as interacções afectivo-sexuais em contextos de viagem turística (Pipa-RN). (dissertação de mestrado) Universidade Federal de Pernambuco, Recife, 2009a.

CANTALICE, T. Feminismo, mercado de sexo e turismo: Reflexões sobre as múltiplas faces e interpretações do sexo mercantil. Bagoas, vol. 2, n. 3, p. 145-178, 2009 b.

CARPAZOO, A. Turismo e identidade: Construção de identidades sociais no contexto do turismo sexual entre alemães e brasileiras na cidade do Recife. (dissertação de mestrado) Universidade Federal de Pernambuco, Recife, 1994.

CARRIER-MOISAN, M.-E. Sexo, namoro e migração: Mudar de vida ou como repensar o turismo sexual no nordeste brasileiro. 26a Reunião Brasileira de Antropologia, 01-04 Junho, Porto Seguro-
Bahia
(Brasil),
2008.
Disponível
em:

<http://www.abant.org.br/conteudo/ANAIS/CD_Virtual_26_RBA/grupos_de_tra

balho/trabalhos/GT\%2035/marie-eve.pdf.> Acesso em: 15 abr. 2013.

CLIFT, S., CALLISTER, C. e LUONGO, M., eds. Gay tourism: Culture, identity, and sex. Londres: Continuum, 2002.

COHEN, E. Transnational marriage in Thailand: The dynamics of extreme heterogamy. In: BAUER, T. e McKERCHER, B., eds. Sex and tourism: journeys of romance, love and lust. Nova Iorque: Haworth Press, p. 57-81, 2003.

COHEN, E. Lovelorn Farangs: The correspondence between foreign men and Thai girls. Anthropological Quarterly, vol. 59, n. 3, p. 115-127, 1986.

COHEN, E. Thai girls and Farang men: The edge of ambiguity. Annals of Tourism Research, 9, p. 403-428, 1982.

COLLINS, D. When sex work isn't 'work': Hospitality, gay life, and the production of desiring labor. Tourist Studies, vol. 7, n. 2, p. 115-139, 2007.

CONSTABLE, N. Pen pals, virtual ethnography, and 'mail order' marriages: Romance on a global stage. Berkeley: University of California Press, 2003.

DAHLES, H. Gigolos and rastamen: Tourism, sex, and changing gender identities. In: em SWAIN, M. e MOMSEN, J., eds. Gender - tourism - fun? Nova Iorque: Cognizant Communication Corporation, p. 180-194, 2002.

DAHLES, H. e BRAS, K. Entrepeneurs in romance: Tourism in Indonésia. Annals of Tourism Research, 26, p. 267-293, 1999.

D'HAUTESERRE, A.-M. Postcolonialism, colonialism, and tourism. In: LEW, A., HALL, C. M. e WILLIAMS, A., eds. A companion to tourism. Oxford: Blackwell Publishing, p. 235-245, 2008.

ENLOE, C. Bananas, beaches and bases: Making feminist sense of the international politics. Berkeley: University of California press, 2000.

FILHO, A. e SARDENBERG, C. O que é que a Bahia tem: Turismo sexual e tráfico de mulheres, Salvador: CHAME/NEIM/UFBA, 1998.

FILHO, A. Fulôs, Ritas, Gabrielas, gringólogas e garotas de programa: Falas, práticas, textos e 
imagens em torno de negras e mestiças, que apontam para a construção da identidade nacional, a partir da sensualidade atribuída à mulher brasileira. (dissertação de mestrado). Universidade Federal da Bahia, Salvador, 1998.

FROHLICK, S. 'I'm more sexy here': Erotic subjectivities of female tourists in the 'sexual paradise' of Caribbean Costa Rica. In: UTENG, T. e CRESSWELL, T., eds. Gendered mobilities. Aldershot: Ashgate, p. 129-142, 2008 .

FROHLICK, S. Negotiating the public secrecy of sex in a transnational tourist town in Caribbean Costa Rica. Tourist Studies, 8, p. 19-39, 2008b.

GRABURN, N. Secular ritual: A general theory of tourism. In: em GMELCH, S., ed. Tourists and tourism: A reader. Long Grove: Waveland Press, p. 23-34, 2004.

GRABURN, N. Tourism and prostitution. Annals of Tourism Research, 10, p. 437-442, 1983.

HALL, C. M. Tourism prostitution: The control and health implications of sex tourism in South-East Asia and Austrália. In: CLIFT, S. e PAGE, S., eds. Health and the international tourist. Londres: Routledge, p. 179-197, 1996.

HEROLD, E., GARCI, R. e DeMOYA, T. Female tourists and beach boys: Romance or sex tourism? Annals of Tourism Research, vol. 28, n. 4, p. 978-997, 2001.

JAACKSON, R. Globalisation and neocolonialist tourism. In: HALL, C. e TUCKER, H., eds. Tourism and postcolonialism: Contested discourses, identities and representations. Abingdon: Routledge, p. 69-183, 2004.

JÚNIOR, C. Trampolim para a vitória. Natal: EDUFRN, 1993.

KEMPADOO, K., ed. Sun, sex, and gold: Tourism and sex work in the Caribbean. Lanham: Rowman \& Littlefield, 1999.

KIBICHO, W. Sex tourism in Africa: Kenya's booming industry. Surrey: Ashgate, 2009.

KING, R. Towards a new map of European migration. International Journal of Population Geography, 8, p. 89-106, 2002.

KRIPPENDORF, J. Sociologia do turismo: Para uma nova compreensão do lazer e das viagens. Rio de Janeiro: Civilização Brasileira, 1989.

KRUHSE-MOUNTBURTON, S. Sex tourism and traditional Australian male identity. In: LANFANT, M.-F., ALLCOCK, J. e BRUNER, E., eds. International tourism: Identity and change. Londres: Sage, p. 192-204, 1995.

LAGUNAS, D. El poder del dinero y el poder del sexo. Antropología del turismo sexual. Perfiles Latinoamericanos, 36, p. 71-98, 2010.

LEHENY, D. A political economy of Asian sex tourism. Annals of Tourism Research, vol. 22, n. 2, p. 367-384, 1995.

LEUNG, P. Sex tourism: The case of Cambodia. In: BAUER, T. e McKERCHER, B. eds. Sex and tourism: Journeys of romance, love and lust. Nova Iorque: Haworth Press, p. 181-195, 2003.

LOWRY, L. Sun, sand, sea \& sex: A look at tourism advertising through the decoding and interpretation of four typical tourism advertisements. Society of Travel and Tourism Educatiors Annual Conference, Miami, Proceedings of Research and Academic Papers, 5, p. 183-204, 1993.

MACHADO, I. Cárcere público: Processos de exotização entre brasileiros no Porto. Lisboa: ICS, 2009.

MANDERSON, L. e JOLLY, M., eds. Sites of desire/economies of pleasure: Sexualities in Asia and the Pacific. Chicago: University of Chicago Press, 1997.

MARQUEZ, A. A 'invisibilidade' de crianças e adolescentes no contexto do turismo sexual em 
Salvador. (dissertação de mestrado). Universidade Federal da Bahia, Salvador, 2009.

McCLINTOCK, A. Imperial leather: Race, gender and sexuality in the colonial contest. Londres: Routledge, 1995.

McKERCHER, B. e BAUER, T. Conceptual framework of the nexus between tourism, romance and sex. In: BAUER, T. e McKERCHER, B. eds. Sex and tourism: Journeys of romance, love and lust. Nova Iorque: Haworth Press, p. 3-17, 2003.

MICHEL, F. Planeta sexo. Turismos sexuais, mercantilização e desumanização dos corpos. Porto: Campo das Letras, 2009.

MIGNOLO, W. Local histories/global designs: Coloniality, subaltern knowledges, and border thinking. New Jersey: Princeton University Press, 2000.

MIN, P. Korean 'comfort women': The intersection of colonial power, gender, and class. Gender \& Society, vol. 17, n. 6, p. 938-957, 2003.

NASH, D. Anthropology of tourism. Nova Iorque: Pergamon Press, 2001.

O’CONNELL-DAVIDSON, J. Sex tourism in Cuba, Race and Class, 1, p. 39-48, 1996.

O'CONNELL-DAVIDSON, J. British sex tourists in Thailand. In: MAYNARD, M. e PURVIS, J., eds. (Hetero)sexual politics. Londres: Taylor \& Francis, p. 42-64, 1995.

OMONDI, R. Gender and the political economy of sex tourism in Kenya's coastal resorts. Paper apresentado no International symposium/doctorial course on feminist perspective on global economic and political systems and women's struggle for global justice. Troms $\varnothing$, Noruega, 24-26 de Setembro, 2003. Disponível em: <www.arsrc.org/downloads/features/omondi.pdf.> Acesso em: 24 mai 2011.

OPPERMANN, M. Sex tourism. Annals of Tourism Research, vol. 26, n. 2, p. 251-266, 1999.

OPPERMANN, M. e McKINLEY, S. Sexual imagery in the marketing of Pacific tourism destinations. In: OPPERMANN, M., ed. Pacific rim tourism. Wellingford: CAB International, p. 116-127, 1997, .

OURIQUES, H. A produção do turismo: Fetichismo e dependência, Campinas: Alínea, 2005.

PARKER, R. Bodies, pleasures and passions: Sexual culture in contemporary Brazil. Boston: Beacon Press, 1991.

PHILLIPS, J. Female sex tourism in Barbados: A postcolonial perspective. Brown Journal of World Affairs, vol. 14, n. 22, p. 201-212, 2008.

PHILLIPS, J. Tourist oriented prostitution in Barbados: The case of the beach boy and the white female tourist. In: KEMPADOO, K., ed. Sun, sex, and gold: Tourism and sex work in the Caribbean. Lanham: Rowman \& Littlefield, p. 183-200, 1999.

PISCITELlI, A., ASSIS, G. e OLIVAR, J., eds.. Gênero, sexo, amor e dinheiro: Mobilidades transnacionais envolvendo o Brasil. Campinas: Unicamp/Pagu, 2011.

PISCITELLI, A. Shifting boundaries: Sex and money in the North-East of Brazil. Sexualities, 10, p. 489-500, 2007.

PISCITELLI, A. Viagens e sexo on-line: A internet na geografia do turismo sexual. Cadernos Pagu, 25, p. 281-327, 2005.

PISCITELLI, A. El tráfico del deseo: Interseccionalidades no marco do turismo sexual no Nordeste do Brasil. Quaderns-e, 4, p. 1-15. 2004a. Disponível em: <http://www.antropologia.cat//antiga/quadernse/04/04_03.htm\#4.> Acesso em: 14 dez. 2011.

PISCITELLI, A. On 'gringos' and 'natives': Gender and sexuality in the context of international sex tourism. Vibrant - Virtual Brazilian Anthropology, vol. 1, n. 1-2, p. 87-114, 2004b. Disponível em: $<$ http://www. vibrant.org.br/issues/v1n1/adriana-piscitelli-on-gringos-and-natives/>. Acesso em: 21 jan. 2011. 
PISCITELLI, A. Entre a praia de Iracema e a União Europeia: Turismo sexual internacional e migração feminina. In: PISCITELLI, A., GREGORI, M. e CARRARA, S., eds. Sexualidade e saberes: Convenções e fronteiras. Rio de Janeiro: Editora Garamond, p. 283-318, 2004c.

PISCITELLI, A. Exotismo e autenticidade: Relatos de viajantes à procura de sexo. In: SILVA, M. C., ed. Outros trópicos - Novos destinos turísticos. Novos terrenos da antropologia. Lisboa: Livros Horizonte, p. 101-123, 2004d.

PRITCHARD, A. e MORGAN, N. Privileging the male gaze: Gendered tourism landscape. Annals of Tourism Research, vol. 27, n. 4, p. 884-905, 2000.

PRUITT, D. e LaFONT, S. For love and money: Romance tourism in Jamaica. Annals of Tourism Research, 22, p. 422-440, 1995.

RAO, N. Sex tourism in South Asia. International Journal of Hospitality Management, vol. 11, n. 2, p. 86-99, 1999.

RIBEIRO, F. B. e SACRAMENTO, O. Sexo, amor e interesse entre gringos e garotas em Natal. Cronos - Revista do Programa de Pós-Graduação em Ciências Sociais da UFRN, vol. 7, n. 1, p. 161$172,2006$.

RIBEIRO, M. et al. Vidas na raia: Prostituição feminina em regiões de fronteira. Porto: Afrontamento, 2007.

ROCA, J. 'Quien lejos se va a casar...': Migraciones (re)negadas. Alteridades, vol. 19, n. 37, p. 133$155,2009$.

ROSALDO, R, Imperialist nostalgia. Representations, 26, p. 107-122, 1989.

RYAN, C. Sex tourism: Paradigms of confusion? In: CLIFT, S. e CARTER, S., eds. Tourism and sex: Culture, commerce and coercion. Londres e Nova Iorque: Pinter, p. 23-40, 2000.

RYAN, C. e HALL, C. M. Sex tourism: Marginal people and liminalities. Londres: Routledge, 2001.

RYAN, C. e KINDER, R. Sex, tourism and sex tourism: Fulfilling similar needs? Tourism Management, vol. 17, n. 7, p. 507-518, 1996.

SACRAMENTO, O. e RIBEIRO, F.B. Trópicos sensuais: A construção do Brasil como geografia desejada. Bagoas, 10, p. 215-232, 2013.

SACRAMENTO, O. From Europe with passion: Frameworks of the touristic male desire of Ponta Negra, in the North-East of Brazil. Current Issues in Tourism, 2015a, DOI: 10.1080/13683500.2015.1056517.

SACRAMENTO, O. Vida boa e história de amor: Desígnios femininos ante turistas europeus no nordeste brasileiro. In: FERNÁNDEZ, Ó., ed. Mujeres en riesgo de exclusión social: Una perspectiva transnacional. Madrid: McGraw-Hill, p. 123-135, 2015 b.

SACRAMENTO, O. Atlântico passional: Mobilidades e configurações transnacionais de intimidade euro-brasileiras. (tese de doutoramento). ISCTE-Instituto Universitário de Lisboa, 2014.

SACRAMENTO, O. Homens em férias abaixo do equador: Questionando a sustentabilidade empírica do conceito de turismo sexual. Actas da International conference on tourism \& management studies, Faro, UALG, II, p. 1189-1192, 2011.

SILVA, A. P. e BLANCHETTE, T. Nossa Senhora da Help: Sexo, turismo e deslocamento transnacional em Copacabana. Cadernos Pagu, 25, p. 249-280, 2005.

SIMONI, V. L'interculturalité comme justification: Sexe 'couleur locale' dans la Cuba touristique. In: LAVANCHY, A., GAJARDO, A. e DERVIN, F., eds. Anthropologies de l'interculturalité. Paris: L' Harmattan, p. 159-187, 2011.

SIMONI, V. Touristic encounters in Cuba: Informality, ambiguity, and emerging relationships. (tese 
de doutoramento) Leeds Metropolitan University, Leeds, 2009.

SMITH, M. e GUARNIZO, L., eds. Transnationalism from below. New Brunswick, NJ: Transaction Publishers, 1998.

SOMMER, D., Fiç̧ões de fundação: Os romances nacionais da América Latina. Belo Horizonte: UFMG, 2004.

TAYLOR, J. Female sex tourism: A contradiction in terms? Feminist Review, vol. 83, n. 1, p. 42- 59, 2006.

TAYLOR, J. Dollars are a girl's best friend? Female tourists' sexual behaviour in the Caribbean. Sociology, 35, p. 749-764, 2001.

TRUONG, T.-D. Sex, money and morality: Prostitution and tourism in Southeast Ásia. Londres: Zed Books, 1990.

TRUONG, T.-D. The dynamics of sex tourism. The case of South-East Ásia. Development and Change, 14, p. 533-53, 1983.

TURNER, V. O processo ritual: Estrutura e anti-estrutura. Petrópolis: Vozes, 1974.

TURNER, L. e ASH, G. La horda dorada. Madrid: Endymion, 1991 [1975].

VEISSIÈRE, S., Ghosts of empire: Sex, mobility, and violence in the transatlantic cultural economy of desire. Berlim: LIT Verlag, 2011.

WONDERS, N. e MICHALOWSKI, R. Bodies, borders, and sex tourism in a globalized world: A tale of two cities - Amsterdam and Havana. Social Problems, vol. 48, n. 4, p. 545-571, 2001.

YEA, S. Former comfort women as touristic objects in South Korea. In: BAUER, T. e McKERCHER, B., eds. Sex and tourism: Journeys of romance, love and lust. Nova Iorque: Haworth Press, p. 139$154,2003$.

\section{Recebido em: 26/05/2016 (1 ${ }^{\mathrm{a}}$ versão $)$}

Aprovado em: 27/07/2016 